\title{
Kunstfehler-Klagen bedrohen gute Medizin
}

\author{
So klagefreudig wie US-amerikanischer Anwälte ist man hierzulande noch nicht, aber ein Blick \\ in die dortige Rechtsprechung schärft das Bewusstsein. Drohen uns bald ähnliche Verhältnisse?
}

_ Die Rechtsprechung in den USA bezüglich ärztlicher Kunstfehler hat die Entschädigungssummen in den vergangenen zehn Jahren exponenziell wachsen lassen und damit dramatischen Einfluss auf die klinische Praxis genommen. Derzeit fallen pro Jahr schätzungsweise 10 Milliarden US-Dollar Entschädigungsgelder in Kunstfehlerprozessen an. Vor allem Ärzte im Notfalleinsatz, die schwere Erkrankungen und ungünstige Entwicklungen rasch mit limitierten diagnostischen Mitteln erkennen müssen, gelten als besonders gefährdete Ärztegruppe für derartige Anklagen.

Am Beispiel der Hodentorsion wurden die Akten der zwischen 2002 und 2013 verhandelten Prozesse analysiert. Die Hodentorsion gilt als besonders problematische Erkrankung, da sie unter der Vielzahl von schmerzhaften Hoden- und Nebenhodenerkrankungen relativ selten ist, die Diagnose nicht immer leicht zu stellen ist und für die adäquate Therapie nur ein schmales Zeitfenster von 4-8 Stunden nach dem Auftreten der Schmerzen besteht.

52 Prozesse konnten ausgewertet werden. $51 \%$ gingen zugunsten des angeklagten Arztes aus, in den anderen wurde der Arzt zur Zahlung einer Entschädigungssumme verurteilt. Notärzte wurden signifikant häufiger verurteilt als Urologen. Daher empfiehlt es sich, bei Skrotalschmerzen frühzeitig einen Urologen zuzuziehen, da diese Maßnahme die Wahrscheinlichkeit einer Verurteilung deutlich reduziert. Eine frühzeitige sonografische Untersuchung der Hoden erwies sich in den Prozessen als keine Garantie für einen Freispruch. Besonders bei sehr jungen Männern ist das
Risiko für eine Verurteilung des erstbehandelnden Arztes hoch. Hier könnten emotionale Gesichtspunkte für die Richter eine Rolle spielen.

- Colaco M, Heavner M, Sunaryo P, Terlecki R. Malpractice litigation and testicular torsion: a legal database review. J Emerg Med. 2015;49:849-54

\section{KOMMENTAR}

Man mag die Entwicklung kritisieren, aber es gibt wohl keinen Ausweg, denn die Klagefreudigkeit und hohe Entschädigungssummen sind ein allgemeiner Trend. Leider fördern sie die Entwicklung einer kostspieligen Defensivmedizin, in der das klinische Urteil und die Wahrscheinlichkeit immer weniger zählen und umfangreiche diagnostische Maßnahmen weniger zum Wohl des Patienten, sondern vor allem zur Abwehr potenzieller Anklagen vorgenommen werden.

Prof. Dr. med. H. S. FüeßI

\section{Die Krätze arbeitet sich durch die Haut}

Eine 61-jährige Frau berichtete über einen seit sechs Monaten bestehenden starken Juckreiz. An Armen, Beinen, Bauch und Rücken fanden sich multiple aufgekratzte Papeln mit honigfarben Krusten (Abb. A). Unter der Verdachtsdiagnose einer Follikulitis und einer Prurigo nodularis wurde über vier Wochen eine Therapie mit Fluocinonid-Creme und Doxycyclin durchgeführt, die aber nichts an den Effloreszenzen än-

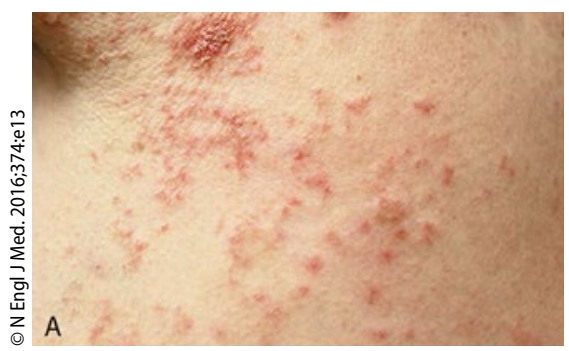

derte. Bioptisch zeigte sich ein eosinophiles Infiltrat, das an ein Arzneimittelexanthem erinnerte. Daraufhin wurden sämtliche Medikamente abgesetzt, was auch zu keiner Besserung führte.

Die Diagnose konnte schließlich mithilfe eines Schabepräparats von den Händen gestellt werden. Unter dem Mikroskop erkannte man eine Scabiesmilbe (Abb. B). Nun wurde topisch mit

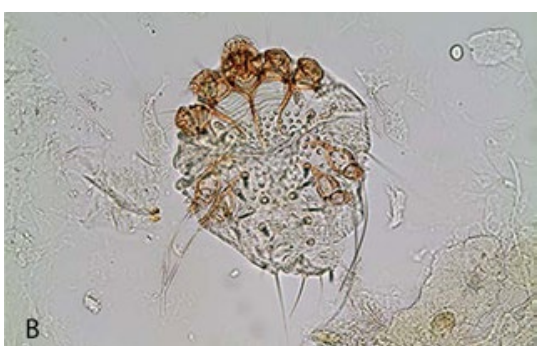

Multiple juckende, aufgekratzte Papeln mit honigfarben Krusten (A); Scabiesmilbe in der mikroskopischen Darstellung (B).

Permethrin-Creme behandelt, worauf sich die Effloreszenzen innerhalb von zwei Wochen zurückbildeten. Typisch für die Scabies ist ein starker Juckreiz, der bei der Bettwärme nachts zunimmt. Ohne Behandlung drohen eine sekundäre Dermatitis aufgrund des Kratzens sowie Impetigo, Ekthyma, Paronychien oder Furunkulose. Die an sich mit bloßem Auge erkennbaren Milbengänge in der Haut können durch Kratzeffloreszenzen und Impetiginisierung maskiert werden. Biopsien zeigen oft unspezifische Veränderungen ohne Milbengänge und Milben selbst, da bei manchen Patienten nur 6-10 Milben im gesamten Integument vorhanden sind. Insofern ist der hier gezeigte Befund als Glückstreffer zu bezeichnen. Mit der gegenwärtigen Migrationswelle wird uns die Scabies wahrscheinlich wieder mehr beschäftigen.

Prof. Dr. med. H. S. FüeßI

- CarrPC, Brodell RT(rbrodell@umc.edu).Scabies. NEngl J Med. 2016;374:e13 